\title{
Margen bruto de diferentes híbridos de espárrago verde (Asparagus officinalis var. altilis L.), en la provincia de Buenos Aires, Argentina
}

\section{Gross margin of different green asparagus hybrids (Asparagus officinalis var. altilis L.) in the province of Buenos Aires, Argentina}

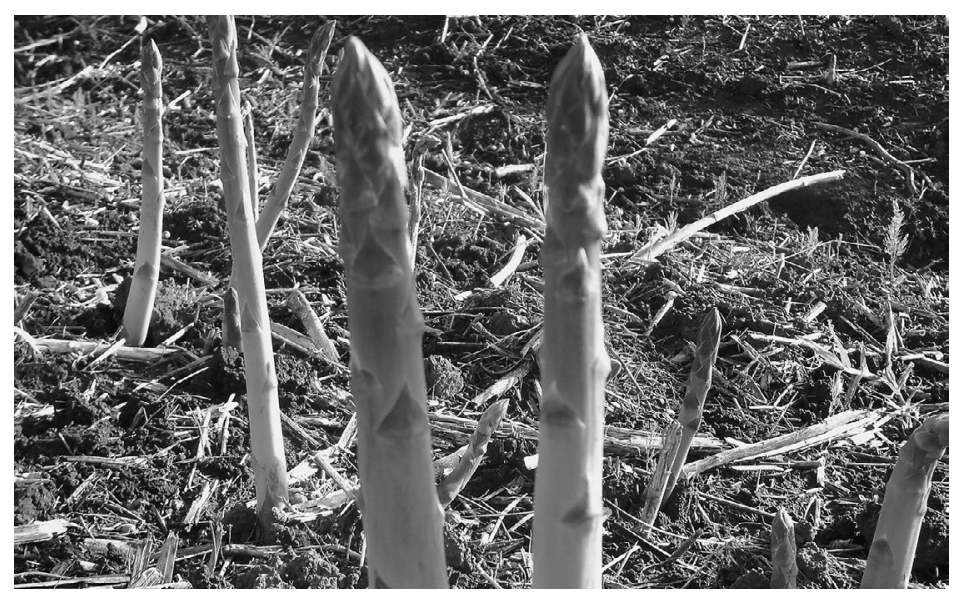

\author{
MARTÍN PASCUALETTI ${ }^{1}$ \\ ANA MARÍA CASTAGNINO1, 2 \\ MARÍA BELÉN ROSINIT \\ MARIO DURANTE 1 \\ ALICIA ZUBIRÍA ${ }^{1}$
}

Turiones de espárrago

emergiendo del suelo, en el

período de cosecha.

Foto: J. Marina

\section{RESUMEN}

El espárrago representa una alternativa productiva para los productores con tendencia creciente a nivel global, cuya demanda se encuentra concentrada en el hemisferio norte, por lo que los países del hemisferio sur, dada su producción en contraestación, tienen la posibilidad de orientar sus producciones tanto a sus propios mercados como a la exportación. Debido a que esta hortaliza presenta gran interacción genotipo/ambiente, es necesario evaluar su productividad y el impacto en los resultados económicos. En tal sentido, el objetivo del presente trabajo es estudiar la incidencia del empleo de diferentes híbridos de espárrago verde en los resultados económicos del cultivo, a través del cálculo de margen bruto, como herramienta en los procesos de decisión de la gestión empresarial, en su cuarto año productivo. A tal fin se evaluó la productividad lograda con siete híbridos enteramente masculinos, de origen italiano: Italo, Zeno, Eros, Ercole, H668, Marte y Giove versus el testigo americano UC-157, en Azul, Buenos Aires, Argentina. El costo total de producción para el año de evaluación fue de US\$5.208. Por ser el espárrago un cultivo perenne se consideró una cuota de amortización que resulto en US\$473. Se obtuvo una productividad comercial promedio de $9 \mathrm{t} \mathrm{ha}^{-1}$, a partir de la cual se elaboró el margen bruto (US\$/ha). El MB promedio fue de US\$/ha 10225,7, con un rango de US\$/ha 8.903,8 (híbrido Marte) y US\$/ha 11.746,3 (híbrido Zeno). Los rendimientos logrados con todos los híbridos en estudio permitieron lograr un margen bruto positivo, lo que determina que se trata de una alternativa productiva alentadora para la región.

Centro Regional de Estudio Sistémico de Cadenas Agroalimentarias (CRESCA), Facultad de Agronomía, Universidad Nacional del Centro de la Provincia de Buenos Aires (UNCPBA), Azul, Argentina

2 Autor para correspondencia.amc@faa.unicen.edu.ar 


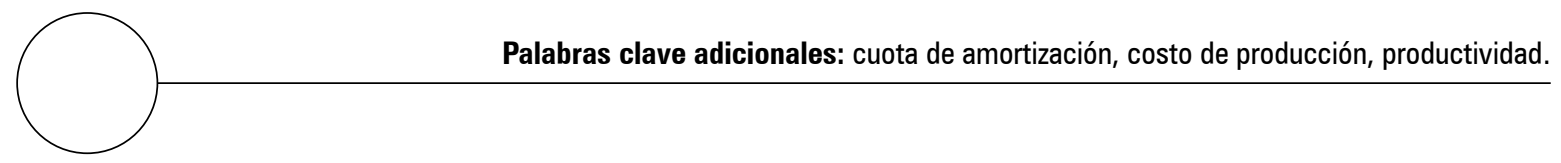

\section{ABSTRACT}

Asparagus represents a productive alternative for farmers that is growing globally. Its demand is centered in the northern hemisphere. Therefore, southern hemisphere countries that produce this crop in the opposite season can market their production in their domestic markets as well as internationally. Since this vegetable has a high genotype-environment interaction, it is necessary to evaluate its productivity and the impact on the economical results. In this sense, the aim of this paper was to study the use incidence of different green asparagus hybrids on the economical results of the crop by means of gross margin calculation as a tool in the decision processes of business management in the fourth year of productivity. The productivity obtained with seven fully male hybrids of Italian origin: Italo, Zeno, Eros, Ercole, H668, Marte and Giove, as compared to the control American UC-157, was evaluated in Azul, Buenos Aires, Argentina. The total production cost for the evaluation year was $\$ 5,208$ USD. As asparagus is a perennial crop, an amortization fee of $\$ 473$ USD was considered. An average commercial productivity of $9 \mathrm{t} \mathrm{ha}^{-1}$ was obtained, on which the gross margin (US\$/ha) was calculated. The GM was 10225.7 US\$/ha with a range between 8903.8 US\$/ha (Marte hybrid) and 11746.3 US\$/ha (Zeno hybrid). The yields obtained with all the studied hybrids allowed for a positive gross margin, demonstrating that it is a promising productive alternative for the region.

Additional key words: amortization fee, production cost, productivity.

Fecha de recepción: 16-09-2013

Aprobado para publicación: 31-10-2013

INTRODUCCIÓN

La diversificación de cultivos en los planteos agrícolas representa una oportunidad a fin de propiciar la optimización de los resultados económicos de dichas empresas. En el caso de las alternativas productivas no tradicionales como el espárrago, resulta imprescindible evaluar la incidencia sobre los resultados económicos de diferentes estrategias tendientes a lograr una rentabilidad que torne viables y atractivas dichas alternativas. El espárrago es una hortaliza perenne, rústica y altamente perecedera, que puede vivir más de 20 años (González, 2006). Requiere del enfoque de cadena y de la optimización de todos los aspectos de manejo, a fin de propiciar la expansión de la superficie cultivada.
Estadísticas de la FAO para el año 2010 han reflejado una producción mundial de 7.832.359,10 t en una superficie cosechada de 1.294.816,50 ha, destacándose en orden de importancia, en producción por continente: Asia 7.082.715,00 t; América 477.229,00 t; Europa 259.620,10 t; Oceanía 9.200,00 t y África $3.595,00 \mathrm{t}$.

La tendencia del comercio mundial se encuentra en crecimiento desde hace dos décadas (Santos, 2011), siendo los principales países productores China, Perú, Alemania, México, Estados Unidos, España, Japón e Italia, en orden de importancia (FAO, 2011). 
Algunos autores afirman que el espárrago fue la hortaliza a nivel global que experimentó el mayor crecimiento en producción (en porcentaje anual) durante el periodo 2000-2005 (7,8 \%), seguida de espinaca (6,5\%), ajo (5,5\%), hongos comestibles $(5,2 \%)$ y lechuga $(4,1 \%)$ (Ferratto y Mondino, 2008; Ferratto et al., 2010).

Desde hace casi un siglo se producen espárragos en Argentina, hortaliza cuya superficie y producción se fue expandiendo hasta la década del noventa cuando llegara a 3.200 ha y un volumen total producido de $8.500 \mathrm{t}$ (1992), con valores de exportación que llegaron a 1.700 t (1997) (Santos, 2011). La tendencia de los últimos años ha sido decreciente tanto en superficie como en producción y en exportaciones. Estas últimas fueron en 2010, 237 t; siendo los principales países de destino Estados Unidos (62\%), Italia (17\%) y Reino Unido (16\%) (Zapata, 2011).

En la actualidad la producción nacional se destina en su mayor parte a cubrir la demanda del mercado interno. Ocho provincias componen el calendario de oferta de la producción nacional, cuya superficie productiva es de 605 ha totales: San Juan: 485,25, Buenos Aires: 66,7; Mendoza: 18,85 Córdoba: 11,2, Catamarca: 11,05, Río Negro: 10,55, Tucumán: 1,05 y Santa Fe: 0,15 ha (Liverotti et al., 2011).

En Argentina, la oferta de espárrago se concentra en los meses de septiembre a noviembre. Debido a una demanda insatisfecha en los meses de febrero, mayo, junio, julio, agosto y septiembre, es necesario recurrir a la importación para satisfacer la demanda, siendo Perú el principal país de origen. Esto hace que el precio del espárrago varíe de US\$3 en noviembre, en plena cosecha Argentina, a US\$12 en marzo, tiempo de importación (Liverotti, 2011).

El espárrago es una planta que se adapta bien a una amplia gama de condiciones climáticas, sin embargo prefiere climas moderadamente frescos con temperatura media anual entre los 15 y los $20^{\circ} \mathrm{C}$. Es una especie que requiere de una estación cálida para crecer y acumular reservas y una estación fría para que la planta entre en reposo invernal. En el caso de "Azul", el promedio de temperatura de la estación cálida es $22^{\circ} \mathrm{C}$, mientras que en invierno, durante el periodo de receso es de $8^{\circ} \mathrm{C}$.

Es una de las pocas especies hortícolas dioicas, cuyas plantas femeninas son menos productivas, pero producen turiones de mayor calibre, mientras que las plantas masculinas son más productivas y producen turiones más finos (Cattivelo, 2002). Actualmente existen en el mercado híbridos enteramente masculinos, cuya producción es más uniforme respecto a los heterocigotas tradicionales, dado que por la interacción genotipoambiente, no siempre mantienen la producción en el tiempo. Tal es el caso del UC-157, tradicionalmente cultivado en Argentina, y que ha sido utilizado en este ensayo como testigo.

En el caso del empleo para el inicio de nuevas plantaciones, de genotipos enteramente masculinos es necesario evaluarlos a lo largo de varios años, a fin de conocer su adaptación a cada zona productiva en particular.

Si consideramos que el ciclo de vida del espárrago puede ser subdividido en las siguientes fases: 1) crecimiento temprano (primeros dos años) caracterizados por un fuerte desarrollo vegetativo; 2) productividad creciente ( $3-4^{\circ}$ año) que corresponde a los dos primeros años de cosecha; 3) productividad estable (4-12 ${ }^{\circ}$ año), y 4) productividad decreciente (12-20 año) (Falavigna, 2004 y 2006), se puede decir que la presente evaluación se realizó en la etapa de productividad creciente.

Al tratarse de un cultivo perenne es muy importante la búsqueda de alternativas tendientes a optimizar la rentabilidad, siendo actualmente la tendencia en países del hemisferio sur, la realización de plantaciones atendiendo a las demandas de los mercados internacionales de 
destino (Paske, 1996). Para el mercado estadounidense se buscan turiones de bajo calibre, mientras que la Unión Europea demanda turiones de gran calibre.

Al introducir híbridos nuevos para la región, se debe estudiar la respuesta a diferentes climas y sistemas de manejo, ya que son características importantes en un híbrido de espárrago, y al mismo tiempo difíciles de predecir (Ellison, 1986). La productividad de los híbridos y la calidad de los turiones debe ser estimada luego de 3 años de controles productivos (considerando 2 años para la entrada en producción y tres de evaluación) en pruebas comparativas, esto se debe, a que no siempre existe una relación directa entre la adaptabilidad al ambiente y la productividad en los primeros dos años de cosecha, ya que algunos genotipos inicialmente, muy productivos pueden no serlo con posterioridad. Como testigo deben incluirse híbridos o variedades de los cuales se conozcan las características productivas y cualitativas (Falavigna, 2001).

En alternativas productivas como la presente, es importante el empleo de herramientas como el cálculo de margen bruto, en los procesos de decisión en la gestión empresarial, a pesar de ser un resultado económico parcial; ya que expone comparativamente la diferencia existente entre el ingreso bruto (IB) y el costo directo (CD) asociado a la actividad, que en este caso particularmente, es elevado. Para el cálculo de dicho margen bruto, en el caso de esta especie, es necesario considerar una cuota de amortización correspondiente al periodo improductivo, dado que se trata de un cultivo perenne que requiere de dos años para la entrada en producción.

El objetivo del presente trabajo es determinar cómo incide en los resultados económicos del cultivo de espárrago verde, el empleo de diferentes híbridos, en su cuarto año productivo, a través del cálculo de margen bruto.

\section{MATERIALES Y MÉTODOS}

\section{Características del ensayo y su evaluación productiva}

El ensayo se llevó a cabo en la Chacra Experimental de la Facultad de Agronomía (UNCPBA), Azul, Provincia de Buenos Aires, situada sobre

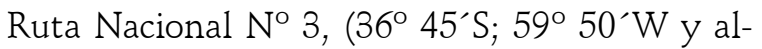
titud $132 \mathrm{msnm}$ ), sobre un suelo Argiudol típico sin impedimentos en el perfil y con $3,55 \%$ de materia orgánica. La zona de estudio es de clima templado, con temperatura media anual de $15^{\circ} \mathrm{C}$, con promedios en verano y en invierno de 22 y $8^{\circ} \mathrm{C}$ respectivamente y precipitaciones promedio de $960 \mathrm{~mm}$, distribuidos uniformemente.

Los híbridos evaluados fueron: enteramente masculinos de origen italiano: Italo, Zeno, Eros, Ercole, H668, Marte y Giove; utilizándose como testigo UC-157, de origen norteamericano.

La superficie total del ensayo fue de $1.200 \mathrm{~m}^{2}$, considerando un ancho total de $24 \mathrm{~m}$ (que incluye 10 surcos a $1,4 \mathrm{~m}$ entre surcos y $5 \mathrm{~m}$ a ambos lados de caminos); y un largo de $50 \mathrm{~m}$ (correspondiente a $7 \mathrm{~m}$ de largo de cada uno de los 4 bloques, 3 caminos internos de $4 \mathrm{~m}$ cada uno y $5 \mathrm{~m}$ de cabeceras en ambos lados). La superficie real de evaluación fue de $392 \mathrm{~m}^{2}$, considerando la superficie de los cuatro bloques de cultivo. La superficie de cada parcela es de $10 \mathrm{~m}^{2}$, con un largo $7 \mathrm{~m}$ y un ancho de 1,4 $\mathrm{m}$.

El diseño experimental utilizado es de bloques al azar, con cuatro repeticiones.

La fecha de plantación fue el 22-11-2006. El marco de plantación fue de 1,4 m entre líneas y 0,3 m entre plantas, o sea una densidad de 23.809 plantas/ha. La profundidad de plantación: 0,25 m.

Antes de la plantación se realizó una fertilización de fondo en banda con fosfato diamónico y posteriormente en cobertura durante el verano 
se efectuaron aplicaciones parcializadas de urea en banda, con dosis acorde a cada momento del ciclo de cultivo, tal como consta en las tablas correspondientes.

El lote se mantuvo libre de malezas mediante labores mecánicas con motocultivador, manuales y químicos: el primer año con Linurón y a partir del segundo desde la plantación con Metribuzín y Pendimetalín, en mezcla en preemergencia (Falavigna, 2004), en las dosis indicadas en el cálculo del margen bruto según la edad de la plantación.

Se regó el año de la plantación por surco y los dos siguientes por goteo, posteriormente el cultivo se manejó en secano.

Entre las características manifestadas para los híbridos en evaluación, en su lugar de origen se destacan (Falavigna, 2001, 2004):

- Italo: primer híbrido íntegramente masculino producido en Italia adaptado a ambientes cálidos y áridos del Mediterráneo, de turiones muy homogéneos con cerrado óptimo de brácteas y que ha manifestado alto vigor en las plantas en terrenos infectados con Fusarium.

- Zeno: híbrido masculino, con esfumaduras antociánicas de calibre medio, adaptado a la producción como verde y blanco, aunque mejor adaptado a blanco.

- Eros: enteramente masculino, caracterizado por elevada productividad en su zona de origen (norte de Italia), de precocidad media, empleado tanto para la producción de turiones verdes como blancos. Presenta un elevado calibre, intensa coloración antociánica y elevada dimensión de las brácteas que permanecen adheridas a la punta del turión, aun cuando superan los $20 \mathrm{~cm}$ de altura.

- Ercole: caracterizado por su elevada productividad, aunque más precoz respecto de Eros, de color verde brillante.
- H668: híbrido experimental que al momento de la plantación aún no era comercial.

- Marte: híbrido enteramente masculino, que presenta una elevada uniformidad fenotípica. De productividad media, de calibre medio y uniforme, adaptado a la producción de turiones blancos y verdes. De color de fondo verde oscuro y brácteas violáceas bien cerradas.

- Giove: híbrido enteramente masculino caracterizado por su elevado calibre y tendencia a buen cierre de brácteas.

- UC-157: híbrido heterocigota, muy precoz, con turiones de calibre medio, con brácteas cerradas, aún en condiciones de cosecha con altas temperaturas. Tradicionalmente cultivado en Argentina, Colombia, Perú, Ecuador y Chile. Ha demostrado gran adaptación, altos rendimientos y una producción temprana de turiones uniformes en color y tamaño (Perfetti Del Corral, 2007).

La cosecha se efectuó en el periodo 19-09-2011 al 16-11-2011, con una frecuencia de día por medio, coincidentemente con la frecuencia utilizada desde el primer periodo de evaluación hasta la fecha. Se cosecharon todas las parcelas de evaluación, debidamente identificadas. Las borduras fueron cosechadas independientemente y no fueron tenidas en cuenta para la evaluación.

Como indicador de cosecha se tuvieron en cuenta los siguientes parámetros: longitud mínima de $23 \mathrm{~cm}$ y cabeza del turión compacta. Se efectuó manualmente, con cuchillos, a ras del suelo, mediante un corte transversal y neto, evitando el corte a bisel.

La producción lograda se acondicionó en el laboratorio de procesado de la Facultad de Agronomía, siguiendo la metodología indicada en el Protocolo de Calidad de Espárrago Verde generado por la SAGPyA (Resolución-SAGPyA N²49/2007). Además, para la etapa de procesa- 
do también se consideraron las buenas prácticas de manufactura (BPM) para salas de empaque, condiciones para las hortalizas frescas y para envases, descritas en el Código Alimentario Argentino (CAA) (1996).

La secuencia de procesado utilizada para la producción lograda de cada parcela fue: lavado, determinación de peso total, corte, selección por calidad en dos categorías, determinación de peso fresco comercial, calibrado de los turiones comerciales y envasado. Las categorías consideradas fueron: comercial, motivo del presente trabajo y descarte. Se consideraron de categoría comercial los turiones bien desarrollados, de consistencia firme, forma, color, sabor y olor característico, en estado fresco, enteros, limpios, sanos, con cabeza compacta y bien cerrada, libres de pudrición, de defectos mecánicos, manchas o indicios de heladas, sin tallos hundidos y sin floración; libres de daños de plagas, insectos vivos y enfermedades, de cualquier material extraño; sin signos de deshidratación; con yemas terminales y brácteas completamente cerradas, libres de signos de lignificación, rectos, sin curvatura del ápice y de tamaño y calibre uniforme y con una tolerancia máxima de color blanco de $4 \mathrm{~cm}$ desde la base (SAGPyA, 2007).

\section{Evaluación económica}

Se realizó un análisis económico a partir de la productividad comercial: PFC (peso fresco comercial) en $\mathrm{kg} \mathrm{ha}^{-1}$ lograda con los distintos híbridos, confeccionándose el margen bruto $(\mathrm{MB}$ en US\$/ha), según las "Normas para medir los resultados económicos en las empresas agropecuarias" de AACREA (1990) para la producción lograda con cada uno de los híbridos cuyo destino fuera el mercado regional.

Para la elaboración del mismo se consideraron:

- Ingreso neto: determinado según el rendimiento del híbrido en cuestión y el precio promedio de mercado para el periodo de evaluación, tomando como referencia de este último, el promedio de los meses de septiembre a noviembre en el Mercado Central de Buenos Aires (MCBA), principal mercado de referencia argentino, para la temporada de cosecha del año de evaluación. Para todos los híbridos se consideró el mismo precio.

- Costos directos: contemplando todas las erogaciones efectivas que se realizaron durante el ciclo productivo para llevar a cabo la producción de espárrago verde, considerando los siguientes costos:

- Costo de labores anuales.

- Costo de insumos utilizados.

- Costo de asesoramiento profesional.

- Gastos varios.

- Gastos de comercialización.

- Cuota de amortización: tomando en cuenta los costos de labores e insumos utilizados durante el primer y segundo año correspondiente al periodo improductivo, dividido por la vida útil del mismo, estimada en 10 años.

Por tratarse de una plantación efectuada con fines experimentales, no se incluyen algunos costos de los bienes de capital, por ejemplo, tierra, vehículos, maquinaria, equipos, herramientas y materiales utilizados. Por dicho motivo, sería conveniente profundizar el presente estudio incorporando otros conceptos, que no debieran faltar en una plantación con fines comerciales.

En el cálculo de margen bruto realizado, se han incluido algunos servicios (a los que no se consideran insumos ya que se consumen en el proceso de producción), como análisis de suelo y honorario por asesoría técnica. 
Para realizar el cálculo de margen bruto se consideró la cotización del dólar para el periodo en estudio; el valor de la UTA, según la revista Márgenes Agropecuarios (2012) y el valor del jornal, según UATRE (Unión Argentina de Trabajadores Rurales y Estibadores), cuyos valores se informan en la tabla a continuación:

\section{Tabla 1. Valores de referencia considerados para el} cálculo de margen bruto (2012).

\begin{tabular}{|c|c|c|}
\hline Valor & Unidades & US\$ \\
\hline UTA (unidad técnica de arada) & US\$/ha & 37,78 \\
\hline Jornal & 8 horas & 50,17 \\
\hline \multicolumn{3}{|c|}{ Cotización dólar $\$ / U S \$ 4,7$} \\
\hline
\end{tabular}

El valor del jornal considerado es el resultado de la suma del sueldo básico, el SAC (sueldo anual complementario), $10 \%$ de indemnización por vacaciones, más $40 \%$ de contribuciones, correspondientes a: aportes jubilatorios, obra social, y seguro. El mismo fue calculado en función de las indicaciones al respecto sobre remuneraciones, del Régimen de Trabajo Agrario, Ley 26.727, Res. 103/2012 del Ministerio de Trabajo, Empleo y Seguridad Social, Comisión Nacional de Trabajo Agrario, vigente en el ámbito de todo el país.

A continuación se detallan los insumos (agroquímicos), considerados para el cálculo de margen bruto y su precio en dólares (tabla 2).

El ensayo se efectuó en el marco del Programa de Investigación y Transferencia Tecnológica $03 \mathrm{~A}$ / 185 "Cadena Espárrago bajo un enfoque sisté- mico" y del proyecto: 03 / A 185 "Optimización de la cadena de valor del espárrago en su etapa adulta, a partir del cuarto año productivo" (20112013)", correspondiente al Núcleo de Actividades Científico-Tecnológicas "Centro Regional de Estudio de Cadenas Agroalimentarias" (CRESCA), Facultad de Agronomía, UNCPBA.

\section{RESULTADOS Y DISCUSIÓN}

\section{Resultados productivos}

Para la realización del presente cálculo de márgen bruto, se ha tomado como referencia la producción lograda de una plantación de espárrago en su cuarta temporada de evaluación, la que resultó en general alentadora, siendo el promedio de productividad comercial lograda por planta de $0,642 \mathrm{~kg}$ (tabla 3) equivalente a $9 \mathrm{t} \mathrm{ha}^{-1}$ duplicando la media histórica nacional de $4 \mathrm{t} \mathrm{ha}{ }^{-1}$, mientras que el rendimiento bruto total fue de $15,5 \mathrm{t}$ ha $^{-1}$ (Castagnino et al., 2012), con un porcentaje de descarte sobre la producción total del $42 \%$. Comercialmente los híbridos más productivos resultaron Zeno, H668 y Eros, mientras que el menos productivo fue Marte, tal como ocurriera en otra evaluación de tamaños de plantines de dichos híbridos (Castagnino et al., 2013).

Los valores obtenidos se corresponden con los obtenidos por Cueto y Lesnick (1999) quienes lograron rendimientos totales entre 10,8 y $15 \mathrm{t}$ $\mathrm{ha}^{-1}$, y rendimientos comerciales entre 6,8 y $9,8 \mathrm{t}$ ha ${ }^{-1}$, con un ensayo de diversos híbridos de espá-

Tabla 2. Valor de referencia de los agroquímicos utilizados para el cálculo de margen bruto (2012).

\begin{tabular}{|l|l|c|c|}
\hline \multirow{2}{*}{ Fertilizante } & Unsumos & Unidades & US\$ \\
& Urea & US $\$ / \mathrm{t}$ & 700 \\
\cline { 2 - 4 } & PDA & US $\$ / \mathrm{t}$ & 730 \\
\hline \multirow{2}{*}{ Herbicida } & Glifosato & US $\$ / \mathrm{L}$ & 8,35 \\
\cline { 2 - 4 } & Linurón & US $\$ / \mathrm{L}$ & 21,71 \\
\cline { 2 - 4 } & Metribuzín & US $\$ / \mathrm{L}$ & 48,4 \\
\cline { 2 - 4 } & Pendimetalin & US $\$ / \mathrm{L}$ & 23 \\
\hline
\end{tabular}


Tabla 3. Productividad total y comercial, y porcentaje descarte de espárrago verde en el cuarto año productivo (2011).

\begin{tabular}{|c|c|c|c|}
\hline \multicolumn{1}{|c|}{ Híbrido } & $\begin{array}{c}\text { Productividad total } \\
\text { ( } / \text { /planta) }\end{array}$ & $\begin{array}{c}\text { Productividad comercial } \\
\text { (g/planta) }\end{array}$ & $\begin{array}{c}\text { Descarte } \\
\text { (\%) }\end{array}$ \\
\hline Italo & $608 \mathrm{c}$ & $352 \mathrm{bc}$ & 42 \\
\hline Zeno & $737 \mathrm{a}$ & $413 \mathrm{a}$ & 44 \\
\hline Eros & $681 \mathrm{abc}$ & $388 \mathrm{abc}$ & 43 \\
\hline Ercole & $635 \mathrm{bc}$ & $364 \mathrm{abc}$ & 43 \\
\hline H668 & $692 \mathrm{ab}$ & $402 \mathrm{ab}$ & 42 \\
\hline Marte & $605 \mathrm{c}$ & $345 \mathrm{c}$ & 43 \\
\hline UC-157 & $644 \mathrm{bc}$ & $382 \mathrm{abc}$ & 40 \\
\hline Giove & $612 \mathrm{c}$ & $367 \mathrm{abc}$ & 42 \\
\hline Promedio & 652 & 377 & 41 \\
\hline
\end{tabular}

rrago empleando una población de 20.000 plantas/ha, en la misma etapa del ciclo de cultivo.

La producción comercial de dichos híbridos en su primera evaluación fue de aproximadamente el $50 \%$. En orden de importancia, los valores obtenidos en esa primer cosecha fueron: en $\mathrm{kg}$ $\mathrm{ha}^{-1}$, de primera calidad, los híbridos UC-157: 5680 (a); Zeno: 4740 (ab); Ercole: 4620 (bc); Eros: 4640 (c); H668: 4500 (c); Giove: 4160 (d); Italo: 4080 (d) y Marte (3640 (e) (Castagnino et al., 2009).

\section{Resultados económicos}

Dicha productividad permitió lograr un margen bruto promedio de US\$/ha 10.225,7, con un rango de US\$/ha 8.903,8 (híbrido Marte) y US\$/ha 11.746,3 (híbrido Zeno).

A continuación se detallan los costos de implantación, producción y procesado en el año de evaluación hasta llegar al cálculo del mencionado $\mathrm{MB}$.

\section{Costos de implantación}

El costo de las labores de implantación del cultivo fue de US\$1.034, tal como se observa en la tabla 4, en la que se detallan las labores correspondientes al año de la plantación, incluyendo preparación del lote, trasplante y manejo del cultivo durante dicho año.

El monto correspondiente a los insumos utilizados durante el año de implantación fue de US\$ 1.953 , tal como se indica en la tabla 5 , incluyendo el costo de los plantines, su traslado, la fertilización de fondo (previo análisis de suelo) y el control de malezas pre transplante.

Transcurrido el primer año vegetativo, se cortó la parte aérea seca, la misma se retiró del lote, se efectuó fertilización de fondo con fosfato di amónico, se escardilló y se controlaron malezas en preemergencia, trabajos cuyo costo se detalla en la tabla 6. Los mismos también fueron considerados para el cálculo de la cuota de amortización, ya que dicho año fue improductivo.

El monto correspondiente a las labores efectuadas durante el segundo año, improductivo al igual que el primero, fue de US $\$ 1.067$, cuyo detalle se muestra en la tabla 6.

En la tabla 7 se indican los insumos utilizados a partir del segundo año.

\section{Cálculo de cuota de amortización}

Como ya se mencionó, por ser el espárrago un cultivo perenne es conveniente considerar una 
Tabla 4. Costo de labores de implantación del cultivo de espárrago en su primer año.

\begin{tabular}{|l|c|c|c|c|}
\hline \multicolumn{1}{|c|}{ Detalle } & Coef. UTA & Cantidad & UTA/ha & Total US\$/ha \\
\hline Disco doble & 0,50 & 4 & 2,00 & 76 \\
\hline Disco doble con rastra de dientes & 0,55 & 1 & 0,55 & 21 \\
\hline Surcado profundo & 0,80 & 2 & 1,60 & 13 \\
\hline Fertilización nitrogenada & 0,35 & 1 & 0,35 & 13 \\
\hline Fertilización fosforada & 0,35 & 1 & 0,35 & 9 \\
\hline Incorporación (rastra de dientes) & 0,25 & 1 & 0,25 & 23 \\
\hline Transplante & 0,60 & 1 & 0,60 & 502 \\
\hline Mano de obra transplante & - & 10 & - & 38 \\
\hline Aporque & 0,50 & 2 & 1,00 & 251 \\
\hline Control de malezas manual & - & 5 & - & 28 \\
\hline Pulverización & 0,25 & 3 & 0,75 & 1.034 \\
\hline \multicolumn{2}{|r|}{ Total } & & & 13 \\
\hline
\end{tabular}

Tabla 5. Costo de insumos utilizados en el año de implantación.

\begin{tabular}{|c|c|c|c|c|}
\hline Detalle & \$/Unidad & Unidades & \$/ha & Total US\$/ha \\
\hline Análisis de suelo & 100,00 & 1 & 100 & 21 \\
\hline Plantines & 0,27 & 23.700 & 6.399 & 1.361 \\
\hline Flete de plantines & - & & 800 & 170 \\
\hline Urea/t & 2,8200 & 180 & 508 & 108 \\
\hline $\mathrm{PDA} / \mathrm{t}$ & 3,43 & 80 & 274 & 58 \\
\hline Glifosato/L & 39,25 & 7,5 & 294 & 63 \\
\hline Linurón/L & 102,037 & 2 & 204 & 43 \\
\hline Asesoramiento profesional & & - & 600 & 128 \\
\hline \multicolumn{3}{|c|}{ Total } & 9.179 & 1.953 \\
\hline
\end{tabular}

Tabla 6. Detalle de las labores realizadas en el segundo año de implantación.

\begin{tabular}{|l|c|c|c|c|}
\hline \multicolumn{1}{|c|}{ Detalle } & Coef. UTA & Cantidad & UTA/ha & Total US\$/ha \\
\hline Control de malezas manual & - & 15 & - & 153 \\
\hline Fertilización nitrogenada & 0,35 & 1 & 0,35 & 13 \\
\hline Escardillada & 0,50 & 1 & 0,50 & 21 \\
\hline Corte parte área seca & 0,55 & 4 & 0,55 & 201 \\
\hline Retirado manual de parte aérea & - & 1 & 0,35 & 13 \\
\hline Fertilización fosforada & 0,35 & 1 & 0,25 & 9 \\
\hline Incorporación (rastra de dientes) & 0,25 & 4 & 1,00 & 38 \\
\hline Pulverización & 0,25 & & & 1.067 \\
\hline
\end{tabular}


Tabla 7. Costo de insumos y de asesoramiento en el segundo año desde la plantación.

\begin{tabular}{|c|c|c|c|c|}
\hline Detalle & $\$ /$ Unidad & Unidades $(\mathrm{kg})$ & $\$ /$ ha & Total US\$/ha \\
\hline Urea & 2,820 & 200,0 & 564 & 120 \\
\hline FDA & 3,431 & 80,0 & 274 & 58 \\
\hline Glifosato & 39,245 & 7,5 & 294 & 63 \\
\hline Metribuzín 40\% & 227,480 & 2,5 & 569 & 121 \\
\hline Pendimetalín 45,5\% & 108,100 & 2,5 & 270 & 58 \\
\hline Asesoramiento profesional & - & - & 1.200 & 255 \\
\hline \multicolumn{3}{|c|}{ Total } & 3.172 & 675 \\
\hline
\end{tabular}

cuota de amortización, que en este caso resulto de US\$473. La cual se calcula considerando todos los costos de los dos primeros años del cultivo, es decir la inversión necesaria para efectuar la plantación. Teniendo en cuenta una vida útil aproximada del cultivo de 10 años, la cuota de amortización del cultivo surge de dividir esos costos en 10 años, tal como se indica en la tabla 8.

\section{Costo de producción en el año de evaluación}

Los costos de las labores, sumado al de los insumos utilizados y otros costos que se incurren en el año productivo constituyen en conjunto los costos directos de producción.

En la cuarta temporada de cosecha correspondiente a la presente evaluación, el costo total de insumos y labores fue de US\$2.066, correspondiendo US\$384 a los insumos utilizados y US\$1.682 a las labores, tal como se detalla en las tablas 9 y 10 .
En el año de evaluación se fertilizó con fosfato diamónico en el periodo de reposo invernal precosecha y con urea en el periodo vegetativo. Para el control de malezas se utilizaron los siguientes herbicidas: Glifosato durante el periodo de reposo vegetativo y Metribuzín y Pendimetalín en mezcla tanto en precosecha como al finalizar la misma en ausencia de turiones.

Los costos de las labores en el año de evaluación, como se puede observar en la tabla 10, son idénticos a los del segundo año excepto que en este año se incurre en el costo de la cosecha, lo que no sucede en los dos primeros años desde la plantación.

\section{Costos de procesado en el año de evaluación}

Una vez cosechados los turiones, los mismos fueron procesados en fresco, siendo el costo de mano de obra e insumos de envasado y embalaje y electricidad de US\$2.110, tal como se detalla en la tabla 11.

Tabla 8. Cálculo de cuota de amortización del cultivo de espárrago verde.

\begin{tabular}{|l|c|c|c|}
\hline \multicolumn{1}{|c|}{ Inversión plantación } & Año 1 & Año 2 & Total \\
\hline Labores de Implantación (US\$) & 1.034 & 1.067 & 2.101 \\
\hline Insumos (US\$) & 1.953 & 675 & 4.628 \\
\hline Total US\$ & 2.987 & 1.742 & \\
\hline $\begin{array}{l}\text { Amortización anual US\$ } \\
\text { (considerando 10 años) }\end{array}$ & 473 & & \\
\hline
\end{tabular}


Tabla 9. Insumos utilizados en el año de evaluación.

\begin{tabular}{|l|c|r|r|r|}
\hline \multicolumn{1}{|c|}{ Productos } & US\$ & US\$/Unidad & Unidades/ha & Total US\$/ha \\
\hline Urea (t) & 600,00 & 0,60 & $200 \mathrm{~kg}$ & 120 \\
\hline FDA (t) & 730,00 & 0,73 & $80 \mathrm{~kg}$ & 58 \\
\hline Glifosato (L) & 8,35 & 8,35 & $7,5 \mathrm{~L}$ & 63 \\
\hline Metribuzín (L) & 48,40 & 48,40 & $2 \mathrm{~L}$ & 97 \\
\hline Pendimetalín (L) & 23,00 & 23,00 & $2 \mathrm{~L}$ & 46 \\
\hline \multicolumn{4}{|c|}{ Total } \\
\hline
\end{tabular}

Tabla 10. Detalle de las labores realizadas en el año de evaluación.

\begin{tabular}{|l|c|c|c|c|c|}
\hline \multicolumn{1}{|c|}{ Detalle } & Coef. UTA & Cantidad & UTA/ha & Total $\mathbf{( \$ / h a )}$ & Total US\$/ha \\
\hline Desmalezado manual & - & 4 & 201 & 628 & 201 \\
\hline $\begin{array}{l}\text { Fertilización } \\
\text { nitrogenada }\end{array}$ & 0,35 & 2 & 26 & 124 & 26 \\
\hline Escardillada & 0,50 & 1 & 19 & 89 & 19 \\
\hline Corte de parte aérea & 0,55 & 1 & 21 & 98 & 21 \\
\hline $\begin{array}{l}\text { Retirado manual de } \\
\text { parte aérea }\end{array}$ & - & 4 & 201 & 628 & 201 \\
\hline Fertilización fosforada & 0,35 & 1 & 13 & 62 & 13 \\
\hline $\begin{array}{l}\text { Incorporación (rastra } \\
\text { de dientes) }\end{array}$ & 0,25 & 1 & 9 & 44 & 9 \\
\hline Pulverización & 0,25 & 4 & 38 & 178 & 38 \\
\hline Cosecha manual & - & 23 & 1154 & 3611 & 1154 \\
\hline \multicolumn{2}{c}{ Total } & & & 5.462 & 1.682 \\
\hline
\end{tabular}

Tabla 11. Costo de procesado en el año de evaluación (cuarto año).

\begin{tabular}{|l|c|c|c|}
\hline \multicolumn{1}{|c|}{ Detalle } & US\$(Unidad & Unidades & Total US\$/ha \\
\hline Mano de obra (Jornal) & 50,170 & 23 & 1.154 \\
\hline Electricidad & & & 64 \\
\hline Bandas elásticas (kg) & 24,500 & 4 & 98 \\
\hline Bandejas & 0,043 & 11.000 & 668 \\
\hline Etiquetas & 0,032 & 20.000 & 128 \\
\hline Film & 31,900 & 4 & 2.550 \\
\hline \multicolumn{2}{|r}{} \\
\hline
\end{tabular}

En la figura 1 se refleja la incidencia de los insumos de empacado y de la mano de obra, como los ítems más significativos de esta etapa, considerando que el $50 \%$ de la producción comercial se acondicionó en atados y el $50 \%$ en bandejas.
En el año de evaluación, además de los mencionados costos de envasado, se consideran para el cálculo de margen bruto, el costo de asesoramiento profesional y de gastos varios, los que se indican en la tabla 12. 


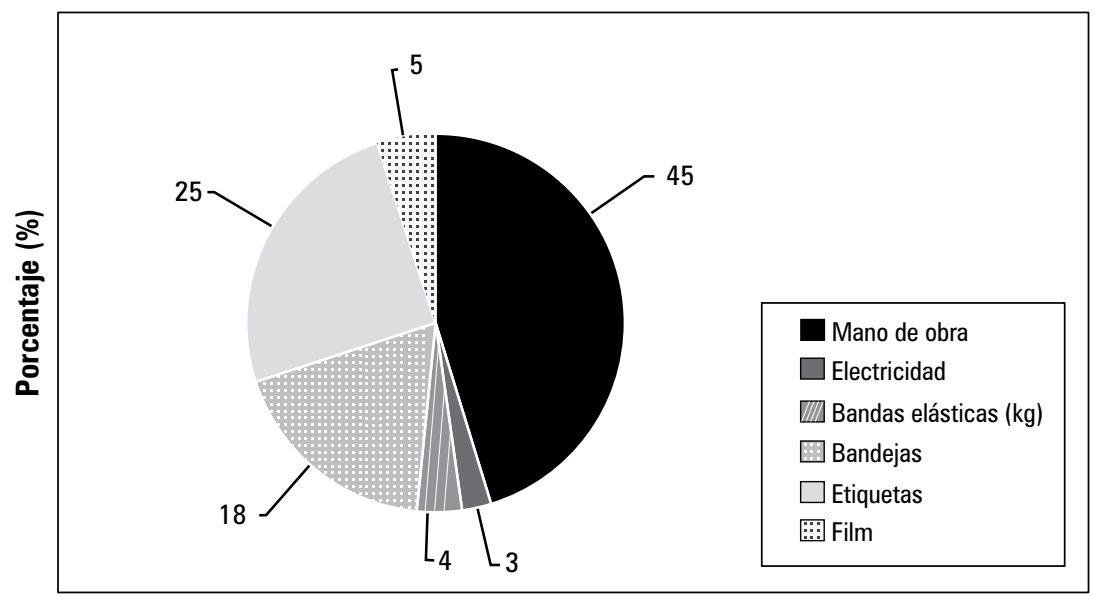

Figura 1. Distribución porcentual de los costos de procesado de espárrago verde.

\section{Tabla 12. Otros costos en el año de evaluación.}

\begin{tabular}{|l|c|}
\hline \multicolumn{1}{|c|}{ Otros costos } & US\$/ha \\
\hline Asesoramiento profesional & 106 \\
\hline Gastos varios & 486 \\
\hline Total & 593 \\
\hline
\end{tabular}

El costo total de producción para el cultivo de espárrago verde a campo considerando la suma del costo de las labores, insumos, procesado y otros costos, fue de US\$5.208, tal como se indica en la tabla 13.

Analizando la distribución porcentual de todos los costos incurridos en el año de evaluación puede observarse que el $50 \%$ corresponde a la etapa productiva y el $50 \%$ al procesado.

\section{Gastos de comercialización}

Los gastos de comercialización de la producción se calcularon teniendo en cuenta un destino regional, teniendo en cuenta que el 50\% fue acondicionado y comercializado en atados de $0,5 \mathrm{~kg}$ y el $50 \%$ en bandejas de $0,45 \mathrm{~kg}$.

Se consideró que tanto las bandejas como los atados fueron embalados en cajas (de $60 \times 40$ x
Tabla 13. Costo total de producción en el año de evaluación.

\begin{tabular}{|l|r|}
\hline \multicolumn{1}{|c|}{ Detalle } & US\$/ha \\
\hline Labores y cosecha & 1.682 \\
\hline Insumos & 384 \\
\hline Procesado & 2.550 \\
\hline Otros costos & 593 \\
\hline Total & 5.208 \\
\hline
\end{tabular}

$20 \mathrm{~cm}$ ), de 16 unidades total, con cuatro niveles de cuatro unidades cada uno. Dichas cajas se ubicaron en pallets, cuya capacidad es de 48 cajas. E1 valor del traslado regional de los pallets es de US\$ 106,38 correspondiente a un recorrido de hasta $300 \mathrm{~km}$. El precio de las cajas fue de US\$/unidad 0,46 . En base a estos datos se obtuvo el valor del gasto de comercialización por kg producido.

\section{Margen bruto de distintos híbridos de espárrago verde}

El margen bruto promedio del cultivo de espárrago verde a campo en su cuarto año productivo fue de US\$/ha 10.226 , considerando que los costos directos fueron de US\$5.208 y el precio promedio fue de US\$2,13, según el MCBA, co- 
rrespondiendo el mayor valor logrado al híbrido Zeno con US\$/ha $11.746,3$, y el menor al híbrido Marte con US $\$ /$ ha $8.903,8$, tal como se muestra en la tabla 14.

Dado que los gastos de comercialización son variables, se calculó el margen de contribución deduciendo el importe de los gastos de comercialización por $\mathrm{kg}$ al precio promedio de comercialización, es decir US\$1,80 (2,13 US\$/ha - 0,35 US\$/kg).
Queda reflejado cómo el rendimiento incide significativamente en el cálculo económico, tal es así que la diferencia de rendimiento entre el híbrido Zeno y Marte permitiría cubrir $66 \%$ de los costos directos. Con respecto a este último hay que considerar que poco se puede hacer para disminuirlo, siendo el costo de procesado el que mayor incidencia tiene en el costo de producción con el 49\%. Los costos de labores, otros costos e insumos representan respectivamente el 32, 12

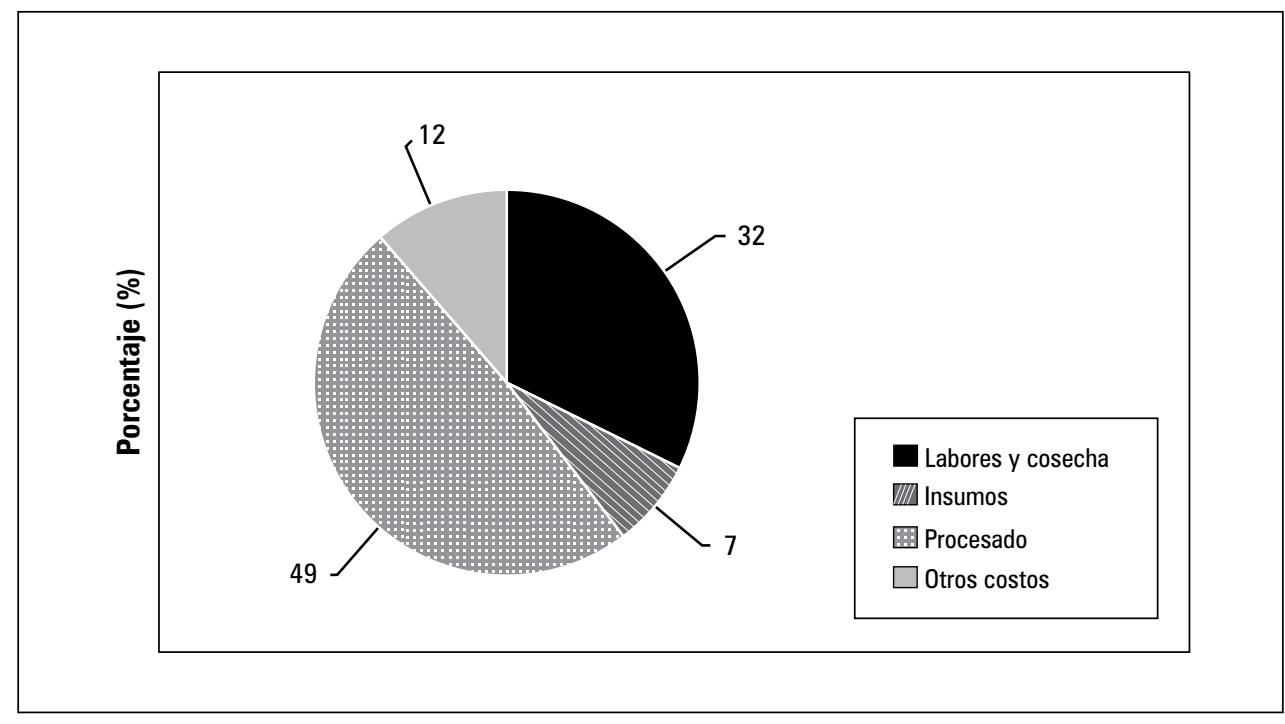

Figura 2. Distribución porcentual de los gastos de producción.

Tabla 14. Margen bruto (US\$/ha) de los híbridos de espárrago verde evaluados.

\begin{tabular}{|c|c|c|c|c|c|c|c|c|}
\hline \multicolumn{9}{|c|}{ Margen bruto: venta mercado interno } \\
\hline Híbrido & Italo & Zeno & Eros & Ercole & $\mathrm{H} 668$ & Marte & UC-157 & Giove \\
\hline Rendimiento $\left(\mathrm{kg} / \mathrm{ha}^{-1}\right)$ & 8.372 & 9.822 & 9.238 & 8.662 & 9.577 & 8.220 & 9.093 & 8.736 \\
\hline Precio promedio (US\$/kg) & 2,1 & 2,1 & 2,1 & 2,1 & 2,1 & 2,1 & 2,1 & 2,1 \\
\hline Ingreso bruto (U\$S) & $17.812,8$ & $20.897,9$ & $19.655,3$ & $18.429,8$ & $20.376,6$ & $17.489,4$ & $19.346,6$ & $18.587,7$ \\
\hline $\begin{array}{l}\text { Gastos de comercialización } \\
\text { (US\$ } / \mathrm{kg} \text { ) }\end{array}$ & 0,4 & 0,4 & 0,4 & 0,4 & 0,4 & 0,4 & 0,4 & 0,4 \\
\hline $\begin{array}{l}\text { Margen de contribución } \\
\text { (US\$/kg) }\end{array}$ & 1,8 & 1,8 & 1,8 & 1,8 & 1,8 & 1,8 & 1,8 & 1,8 \\
\hline Ingreso neto (US\$) & 14.855 & 17.427 & 16.391 & 15.369 & 16.993 & 14.585 & 16.134 & 15.501 \\
\hline Costo producción (US\$/ha) & $5.208,2$ & $5.208,2$ & $5.208,2$ & $5.208,2$ & $5.208,2$ & $5.208,2$ & $5.208,2$ & $5.208,2$ \\
\hline Amortización (US\$/ha) & 472,9 & 472,9 & 472,9 & 472,9 & 472,9 & 472,9 & 472,9 & 472,9 \\
\hline Margen bruto (US\$/kg) & $9.173,5$ & $11.746,3$ & $10.710,1$ & $9.688,1$ & $11.311,6$ & $8.903,8$ & $10.452,6$ & $9.819,8$ \\
\hline
\end{tabular}


y $7 \%$ del costo de producción. Por lo que a la hora de maximizar el beneficio económico debemos apuntar a disminuir el costo de procesado y labores, ya que estos costos representan el $81 \%$ del costo total de producción.

El costo de producción por kg de producto, con amortización es de US\$0,63. Si el gasto de comercialización representa US\$0,35 quiere decir que por cada kg producido, el margen de contribución es de 1,78 US\$/kg.

Considerando los valores mencionados correspondientes al costo total de producción, gastos de comercialización y cuota de amortización, el costo total promedio fue de US\$5.205, siendo el rendimiento de indiferencia de $3.192 \mathrm{~kg} \mathrm{ha}^{-1}$, valor que surge del cálculo indicado en la tabla 15.

\section{Tabla 15. Cálculo del rendimiento de indiferencia.}

\begin{tabular}{|l|c|}
\hline \multicolumn{1}{|c|}{ Detalle } & 8.965 \\
\hline Rendimiento promedio $\left(\mathrm{kg} \mathrm{ha}^{-1}\right)$ & 2,13 \\
\hline Precio de venta $(\mathrm{US} \$ / \mathrm{kg})$ & 0,35 \\
\hline $\begin{array}{l}\text { Gastos de comercialización promedio/ } \\
\mathrm{kg}((\mathrm{US} \$ / \mathrm{kg})\end{array}$ & 1,78 \\
\hline Margen de contribución (US\$/kg) & \\
\hline & 5208,2 \\
\hline Costo producción (US\$/kg) & 472,9 \\
\hline Amortización (US\$/kg) & 5.681 \\
\hline Total costo fijo & \\
\hline & \\
\hline Rendimiento de indiferencia $\left(\mathrm{kg} \mathrm{ha}^{-1}\right)$ & 3.192 \\
\hline US\$5.681 / US\$1,78 & \\
\hline
\end{tabular}

Dicho rendimiento de indiferencia ha sido superado con todos los híbridos evaluados en su cuarta cosecha, lo que demuestra que con cualquiera de ellos es posible obtener un beneficio económico positivo, el que será mayor cuanto más alto sea el rendimiento del hibrido utilizado.

Resulta alentador además que tres de los genotipos italianos en evaluación, superaron al testigo americano, UC-157, también en los resultados económicos logrados.

En muchos casos, cuando las diferencias varietales son muy evidentes, los productos hortícolas reciben precios diferenciales. En el caso de Argentina, para espárrago verde hay un único precio de mercado promedio, que no distingue categorías. Además, en el caso de esta hortaliza, sus diferencias en calidad están dadas por aspectos que benefician directamente al productor, como por ejemplo la mayor uniformidad de calibre en la producción lograda, lo que le permite simplificar el trabajo de poscosecha; el mayor grado de cierre de las brácteas, que le permite minimizar el descarte, etc. En este caso, no se observan diferencias en la calidad externa de los distintos híbridos, por lo que se ha considerado un único precio.

En el cálculo de costos, para emprendimientos comerciales resultaría imprescindible incluir el uso del suelo, como factor de producción, por tratarse el espárrago de un cultivo perenne.

El valor calculado de $\mathrm{MB}$ corresponde al quinto año del cultivo, por lo que, si bien no se debe generalizar para todo su ciclo de vida, puede tomárselo como referencia para todos los años correspondientes a la etapa de productividad estable.

\section{CONCLUSIONES}

El cultivo de espárrago verde, en su cuarto año productivo, con destino al mercado regional como producto fresco, resultó económicamente viable en el centro de la provincia de Buenos Aires para la campaña 2012.

Los rendimientos logrados, con todos los híbridos en estudio permitieron lograr un margen bruto positivo, lo que determina que se trata de una alternativa productiva alentadora para la región.

La productividad comercial lograda de $9 \mathrm{t} \mathrm{ha}^{-1}$ promedio, que duplica la media histórica argen- 
tina (para una esparraguera adulta) podría resultar aún superior, de cosechar con una frecuencia diaria, lo que permitiría lograr un margen bruto superior.

Sería necesario completar el siguiente estudio con un análisis más exhaustivo, especialmente cuando el cultivo llegue a plena producción y considerando nuevos mercados como destino.

Resultaría conveniente complementar el presente estudio con uno más abarcativo, que incluya todos los conceptos de interés para los emprendedores, otros mercados de destino y por un pe- riodo más extenso. Además teniendo en cuenta que la calidad superior de los genotipos italianos masculinos, sería posible acceder a un mejor precio promedio del considerado.

Por su simplicidad de manejo cultural en su etapa adulta, su longevidad, su elevada productividad y los resultados económicos logrados, puede decirse que el cultivo de espárrago verde representa una alternativa interesante de diversificación productiva, para aquellos países que, como Argentina, cuentan con una demanda interna creciente y con posibilidades de comercialización en contraestación.

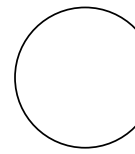

AACREA. 1990. Normas para medir los resultados económicos en las empresas agropecuarias. Convenio CREA-BANCO RIO. Buenos Aires.

Castagnino, A.M., K.E. Díaz, M.B. Rosini y A. Falavigna. 2012. Evaluation of some critical points of the asparagus food chain in Argentina. Acta Hort. 950, 37-51.

Castagnino, A.M., K. Díaz y M.B. Rosini. 2009. Manual de cultivos hortícolas innovadores. Editorial Hemisferio Sur, Buenos Aires.

Castagnino, A.M., K.E. Díaz, M. Rosini, M. Pascualetti, A. Guisolis, A. Novella y A. Falavigna. 2012. Productividad a campo de ocho híbridos de espárrago verde (Asparagus officinalis var. Altilis L.) en su cuarto período de evaluación. p. 309. En: Memorias XXXV Congreso Argentino de Horticultura. Asociación Argentina de Horticultura (ASAHO), 25 09-2012, Corrientes, Argentina.

Castagnino, A.M., M. Pascualetti, M.B. Rosini, K. Díaz, A. Guisolis, J. Marina, A. Falavigna, A. y S. Benson. 2010. Evaluation of Italian all-male asparagus (Asparagus officinalis L.) hybrids and seedling sizes as strategies to optimize green spear production in Argentina. J. Interamer. Soc. Trop. Hort. 57, 1-20.

Cattivelo, C.E. y D. Della. 2002. L'asparago. Bioagricultura, pp. 33-34.

\section{REFERENCIAS BIBLIOGRÁFICAS}

Código Alimentario Argentino. 1996. Buenas prácticas de manufactura. En www.alimentosargentinos. gov.ar/contenido/marco/CAA/capitulospdf/Capitulo_XI.pdf; consulta: septiembre de 2012.

Cotización Dólar. 2012. Cotización histórica del dólar en Argentina. En: http://www.cotizacion-dolar. com.ar/cotizaciones_dolar_historico.php; consulta: septiembre de $20 \overline{1} 2$.

Cueto, G.G. y D.J. Lesnick. 1999. Yield performance of new asparagus cultivars at Dole Tropifresh, Polomolok, Philippines. Acta Hort. 479, 163-168.

Ellison, J.H. 1986. Asparagus breeding. pp. 521-569. In: Bassett, M.J. (ed.). Breeding vegetable crops. Avi Publisher Co., Westport, CT.

Falavigna, A. 2004. Strategie per ottimizzare e valorizzare la produzione di asparago in Sicilia. Programma Interregionale di Ricerca e Sperimentazione Applicata, Trasferimento delle Innovazione agli operatori di Filiera e Programmi a forte contenuto innovativo. Editorial Grillo e Famá, Italia. pp. 15-17.

Falavigna, A. 2006. I Punti critici dell'asparago in campo e nel post-raccolta. Speciale Le strategíe di coltivazione, le esigenze di valorizazzione. L'Informatore Agrario 52, 52-55.

Falavigna, A. y A.D. Palumbo. 2001. La coltura dell' asparago. Editorial Calderini Edagricole, Bologna, Italia. pp. 52-130. 
FAO. 2011. Faostat. En: http://faostat.fao.org/site/291/ default.aspx; consulta: octubre de 2011.

Ferratto, J. y M.C. Mondino. 2008. Producción, consumo y comercialización de hortalizas en el mundo. Revista Agromensajes, p. 24.

Ferratto, J.A. y F. Rodríguez. 2010. Buenas prácticas agrícolas para la agricultura familiar. Cadena de las principales hortalizas de hojas en Argentina. Organización de las Naciones Unidas para la Agricultura y la Alimentación (FAO), Ministerio de Agricultura, Ganadería y Pesca de la Nación Argentina, Instituto Nacional de Tecnología Agropecuaria (INTA) y Universidad Nacional de Rosario, Buenos Aires.

González C., M. L. 2006. Utilización del cultivo de espárrago verde en bandas para el control de la erosión en terrenos de mediana pendiente. Dirección General de Desarrollo Rural, Centro de Transferencia Agroalimentaria, Publicación No. 174. Unión Europea, Diputación General de Aragón, Argentina.

Liverotti, O., A. Zubiría y A. Castagnino. 2011. Tendencias de la comercialización de espárrago en el MCBA durante el último bienio. En: XXXIV Congreso Argentino de Horticultura. Asociación Argentina de Horticultura (Asaho), Buenos Aires.

Mercado Central.2012. Precio de hortalizas. En http:// www.alcentral.com.ar/precios.html; consulta: noviembre de 2012 .
Paske, M.R.A. 1996. Importing fresh asparagus - A personal viewpoint. Acta Hort. 415, 19-24.

Perfetti Del Corral, J. 2007. Espárrago: Producción y comercio. Corporación Colombia Internacional (Bogotá). Serie Técnica No. 3. pp. 7-9.

Revista Márgenes Agropecuarios. 2012. Valor y coeficientes UTA. En http://www.margenes.com; consulta: junio de 2012.

SAGPyA. 2007. Secretaría de Agricultura, Ganadería, Pesca y Alimentos 2007. Protocolo de calidad para espárrago fresco. Resolución SAGPyA No 249/2007. En: http://www.alimentosargentinos.gov.ar/programa_calidad/diferenciacion/sello/SAA010_Esparrago_v08.pdf; consulta: septiembre de 2013.

Santos, B. 2011. Análisis económico del empleo de dos híbridos (UC-157 y Italo) de espárrago verde con destino a diferentes mercados. Trabajo final. Facultad de Ciencias Agrarias, Universidad Católica Argentina, Buenos Aires.

UATRE. 2012. Escala salarial de los trabajadores rurales. En: http://www.uatre.org.ar/salario; consulta: septiembre de 2012.

Zapata, C. 2011. Comercialización externa del espárrago, en Argentina. Taller Situación y Perspectivas del Cultivo de Espárragos. XXXIV Congreso Argentino de Horticultura, 29-09-2011, Buenos Aires. 